\title{
On the Kernel and the Nuclei of 8-Dimensional Locally Compact Quasifields
}

\author{
By \\ Thomas Buchanat and Hermany HäHL
}

1. Introduction. In this note we determine the nuclei of 8-dimensional real nonassociative division algebras and also study the corresponding substructures of 8-dimensional locally compact quasifields.

1.1. The left, middle or right nucleus of a (not necessarily associative) division algebra $D$ is defined to be the (skew) subfield consisting of those elements which associate with all elements of $D$ from the left, middle or right respectively (cf. 1.3 below). A consequence of the theorems of this paper is the

Corollary. If $D$ is an 8-dimensional real division algebra with a unit, then the left nucleus, the middle nucleus and the right nucleus of $D$ consist solely of the real scalar multiples of the unit element. In particular, all nuclei of $D$ are isomorphic to the field $\mathbb{R}$ of real numbers.

In contrast, there exist real division algebras of dimension four with nuclei isomorphic to the complex field $\mathbb{C}$ - examples are contained in [9] - and, of course, all three nuclei of the quaternion field $\mathbb{H}$ are $\mathbb{H}$ itself.

Actually, the corollary itself can be established directly and easily by applying a result in algebraic topology due to $A$. Borel and J.-P. Serre $[2,17.1]$. (See the proof of Theorem 1 and the discussion in section 1.4 below for details.) The BorelSerre result, however, cannot be directly applied to determine the nuclei of more general structures. Moreover, the proof of the Borel-Serre result as given in [2] makes use of heavy machinery of algebraic topology, and we can establish our corollary with more elementary techniques which involve only the homotopy theory of classical groups as expounded in Steenrod [18]. This is essentially done in the proof of Theorem 2 below.

Our motivation for the study of the kernel and the nuclei in division algebras and quasifields was the fact that these substructures are related to certain groups of axial collineations in the affine planes coordinatized by these domains. For the details of this relationship we refer the reader to Dembowski [5, 3.1.28 and 3.1.30].

1.2. In $[14, \S 7]$ H. Salzmann describes some of the basic properties of a locally compact quasifield $Q$ of dimension $n(n \geqq 1$ ). The structure of $Q$ can be characterized 
as being the real vector space $\mathbb{R}^{n}$ together with a continuous multiplication

$$
\mathbb{R}^{n} \times \mathbb{R}^{n} \rightarrow \mathbb{R}^{n}:(x, y) \mapsto x \circ y
$$

which satisfies the following axioms:

(Q 1) The zero vector $0 \in \mathbb{R}^{n}$ is a zero for 0 .

(Q 2) There exists a two-sided unit 1 for 0.

(Q 3) For every $a, b \in \mathbb{R}^{n}$ with $a \neq b$ the map $\mathbb{R}^{n} \rightarrow \mathbb{R}^{n}: x \mapsto a \circ x-b \circ x$ is linear and nonsingular.

(Q4) For every $a, c \in \mathbb{R}^{n}$ with $a \neq 0$ there exists a solution to the equation $y \circ a=c$ (which by axiom (Q 3) is unique); this solution is required to depend continuously on the pair $(a, c)$.

As noted in $[14,7.12]$, J. F. Adams's theorem on the Hopf invariant implies that the only possible dimensions of such quasifields are 1, 2, 4 and 8 . An $n$-dimensional real division algebra is just an $n$-dimensional locally compact quasifield with both distributive laws. Section 2 below contains examples of 8-dimensional locally compact quasifields which are not division algebras.

The kernel $K$ of $Q$ is defined to be the set of elements which associate and distribute with all elements of $Q$ from the right:

$$
K=\left\{a \in \mathbb{R}^{n} \mid \wedge x, y \in \mathbb{R}^{n}: \begin{array}{l}
(x \circ y) \circ a=x \circ(y \circ a) \text { and } \\
(x+y) \circ a=x \circ a+y \circ a
\end{array}\right\} .
$$

In the special case of a division algebra the kernel coincides with the right nucleus.

It is easily verfied that the kernel is a topological field with respect to vector space addition and the multiplication 0 . By continuity the real scalar multiples of the unit element form a central subfield of $K$ isomorphic to $\mathbb{R}$. Thus $K$ may be considered as a real associative division algebra. By a well-known theorem of Frobenius $K$ is isomorphic to one of the topological fields $\mathbb{R}, \mathbb{C}$ or $\mathbb{H}$. In the 8-dimensional case we prove more specifically:

Theorem 1. The kernel of an 8-dimensional locally compact quasifield is isomorphic to $\mathbb{R}$.

1.3. The left nucleus $N_{\lambda}$ and the middle nucleus $N_{\mu}$ of $Q$ are defined as in a division algebra, namely as

$$
\begin{aligned}
& N_{\lambda}=\left\{a \in \mathbb{R}^{n} \mid \wedge x, y \in \mathbb{R}^{n}:(a \circ x) \circ y=a \circ(x \circ y)\right\}, \\
& N_{\mu}=\left\{a \in \mathbb{R}^{n} \mid \wedge x, y \in \mathbb{R}^{n}:(x \circ a) \circ y=x \circ(a \circ y)\right\} .
\end{aligned}
$$

The nuclei $N_{\lambda}$ and $N_{\mu}$ are not necessarily closed under addition. (See the examples in section 2 below.) However, $N_{\lambda}^{\times}=N_{\lambda} \backslash\{0\}$ and $N_{\mu}^{\times}=N_{\mu} \backslash\{0\}$ are topological groups with respect to the multiplication o (the verification of this fact is left to the reader). By identifying each element $a \in N_{\lambda}^{\times}$with the linear map $\mathbb{R}^{n} \rightarrow \mathbb{R}^{n}: x \mapsto a \circ x$ we obtain an isomorphism of topological groups mapping $N_{\lambda}^{\times}$onto a subgroup of $G L(n, \mathbb{R})$; this subgroup is closed because of axiom ( $\mathrm{Q} 2)$ and because of the continuity 
of $\circ$. Analogous statements hold for the multiplicative group $N_{\mu}^{\times}$of the middle nucleus. Consequently, $N_{\lambda}^{\times}$and $N_{\mu}^{\times}$are both Lie groups. We shall prove

Theorem 2. If $N_{\lambda}^{\mathrm{x}}$ is the multiplicative group of the left nucleus of an 8-dimensional locally compact quasifield, then $N_{\lambda}^{\times}$is either 1) finite, 2) discrete and isomorphic to a semidirect product of a finite normal subgroup with $\mathbb{Z}$, or 3 ) isomorphic as a topological group to the direct product of a finite group and the multiplicative group $\mathbb{R}_{\text {pos }}$ of the positive real numbers. The elements $a \in N_{\lambda}^{\times}$of infinite order induce linear maps $x \mapsto a \circ x$ with determinant different from 1. These assertions hold for the multiplicative group $N_{\mu}^{\times}$ of the middle nucleus as well.

In all cases $N_{\lambda}^{\times}$(and $N_{\mu}^{\times}$) contains a largest compact subgroup $F$, and this subgroup is actually finite. By left multiplication $F$ can be considered as a finite fixed point free linear group on $\mathbb{R}^{8}$. Up to equivalence a complete list of all such groups can be found in Wolf $[20$, p. 225]. Further restrictions on $F$ can be obtained by applying Folkman's results [6] on equivariant maps of spheres into the classical groups (cf. [3]). However, a complete determination of $F$ using this approach seems to be inextricably tied up with the unsolved problem of determining the homotopy class of the map $\lambda: \mathbb{R}^{8} \backslash\{0\} \rightarrow G L(8, \mathbb{R}): a \mapsto \lambda_{a}$ with $\lambda_{a}(x)=a \circ x$ which is associated with the quasifield multiplication $\circ$. If one assumes that $\lambda$ is homotopic to the corresponding map of the classical Cayley algebra $\mathbb{O}$ or its opposite algebra $\mathbb{O}$ op as is generally conjectured, Folkman's results can be used to show that $F$ has at most two elements.

1.4. It is immediate that the Corollary in 1.1 above is a consequence of Theorems 1 and 2. Actually, this Corollary follows from each of them separately. In fact, by reversing the order of the factors in the multiplication of a division algebra $D$ (that is by passing from $D$ to the opposite algebra $D^{\circ}$ ) the roles of the left nucleus and the kernel are interchanged; and a similar switch involving the left and middle nuclei is obtained by transposition (see [4, §2] and [13, Prop. 4 and Prop. 5(3)]).

1.5. A weak nucleus $W$ of a division algebra $D$ is a (skew) subfield of $D$ such that the equation $a \circ(b \circ c)=(a \circ b) \circ c$ holds provided two of the three elements $a, b, c$ of $D$ lie in $W$. For example, any two-dimensional subfield of the Cayley algebra $\mathbb{O}$ is a maximal weak nucleus of $\mathbb{O}$.

If $D$ is a real $n$-dimensional division algebra, then the topological closure in $D$ $\left(=\mathbb{R}^{n}\right)$ of a weak nucleus is obviously a weak nucleus too. The following theorem may be of algebraic interest:

Theorem 3. A topologically closed weak nucleus of an 8-dimensional real division algebra is isomorphic either to $\mathbb{R}$ or to $\mathbb{C}$.

2. Examples. The following examples illustrate some of the diverse forms taken on by nuclei of 8-dimensional locally compact quasifields. 
2.1. In what follows, we identify the Cayley numbers 10 with the real vector space $\mathbb{R}^{8}$. Ordinary multiplication and inversion are understood to be those of $\mathbb{O}$.

Let $\varphi: \mathbb{R}_{\text {pos }} \rightarrow \mathbb{O}$ be a fixed continuous map from the positive reals into $\mathbb{O}$ such that $\varphi(1)$ is the unit of $\mathbb{O}$ and $|\varphi(r)|=r$ for all $r \in \mathbb{R}_{\text {pos }}$. We define a new multiplication $\circ$ on $\mathbb{R}^{8}$ which depends on $\varphi$ by

$$
a \circ x=\left\{\begin{array}{cc}
\left(\left(a \cdot \varphi(|a|)^{-1}\right) x\right) \cdot \varphi(|a|) & \text { for } a \neq 0 \\
0 & \text { for } a=0 .
\end{array}\right.
$$

The reader can easily verify that o satisfies the axioms ( 1 1) through ( $Q 4)$. Note that $\circ$ is also norm preserving, i.e. $|a \circ x|=|a||x|$. In general with respect to $\circ$ and vector addition only one distributive law holds. The definition of $\circ$ is analogous to the multiplication in 4-dimensional near fields ([11], [19]).

To describe the nuclei $N_{\lambda}$ and $N_{\mu}$ of the quasifields with these multiplications we distinguish three cases.

Case 1: $\varphi$ is a multiplicative homomorphism.

Then

$$
N_{\lambda}^{\times}= \pm \varphi\left(\mathbb{R}_{\text {pos }}\right) \quad \text { and } \quad N_{\mu}^{\times}=\left\{ \pm \frac{\varphi(r)^{2}}{r} \mid r \in \mathbb{R}_{\text {pos }}\right\} \text {. }
$$

For example, if $\varphi$ is the homomorphism $\varphi(r)=r e^{i \alpha \cdot \log r}$ with a fixed real $\alpha \neq 0$, then neither $N_{\lambda}$ nor $N_{\mu}$ are the one-dimensional subspace spanned by the unit, nor is $N_{\lambda}=N_{\mu}$.

Case 2: $\varphi$ is not multiplicative, but the image of $\varphi$ is contained in a two-dimensional subfield of $\mathbb{O}$.

Then either the set

$$
J=\left\{r>1 \mid \wedge s \in \mathbb{R}_{\text {pos }}: \varphi(r s)=\varphi(r) \varphi(s)\right\}
$$

is empty, in which case

$$
N_{\lambda}^{\times}=N_{\mu}^{\times}=\{1,-1\} \text {. }
$$

or $J$ has a smallest element $r_{0}$; then

$$
N_{\lambda}^{\times}=\left\{ \pm \varphi\left(r_{0}\right)^{n} \mid n \in \mathbb{Z}\right\} \text { and } N_{\mu}^{\times}=\left\{ \pm\left(\frac{\varphi\left(r_{0}\right)^{2}}{r_{0}}\right)^{n} \mid n \in \mathbb{Z}\right\} \text {. }
$$

Case 3: The image of $\varphi$ is not contained in a two-dimensional subfield of $\mathbb{O}$.

Then either the set

$$
J \cap \varphi^{-1}(\mathbb{R})=\left\{r>1 \mid \wedge s \in \mathbb{R}_{\text {pos }}: \varphi(r s)= \pm r \cdot \varphi(s)\right\}
$$

is empty, in which case

$$
N_{\lambda}^{\times}=N_{\mu}^{\times}=\{1,-1\},
$$

or $J \cap \varphi^{-1}(\mathbb{R})$ has a smallest element $r_{1}$; then

$$
N_{z}^{\times}=N_{\mu}^{\times}=\left\{ \pm r_{1}^{n} \mid n \in \mathbb{Z}\right\} .
$$


The proofs consist of arithmetical calculations in $\mathbb{1}$ using the Moufang identities. We shall not reproduce them here.

2.2. Further examples of 8-dimensional locally compact quasifields can be obtained as follows. Consider the affine plane over a real 8-dimensional division algebra different from the alternative field $\mathbb{0}$. Choose a frame of reference for this plane with coordinate axes nonparallel to the original ones. The coordinate domain with respect to this frame is an 8-dimensional locally compact quasifield ([14, §7]). Explicit formulae for this change of coordinates are given in Skornyakov [16]. Both the left and middle nuclei of a quasifield obtained in this fashion consist of 0 and 1 only, since any nontrivial element of either nucleus would induce a collineation which would move the shears direction, contradicting a well-known theorem of Skornyakov ([17], see also [1, Hilfssatz 4 and 5] and [10, Theorem 6.16 p. 140]) together with the fact that $\mathbb{O}$ is the only 8-dimensional alternative field over $\mathbb{R}([14,7.27])$.

Note that the examples of 2.1 above always have -1 in their nuclei and thus are not of the type we have just described.

\section{Proofs of the theorems.}

Proof of Theorem 1. Let us assume the theorem is false. Then there is an 8-dimensional locally compact quasifield $Q$ whose kernel $K$ is isomorphic to $\mathbb{C}$ or to $H$. In both cases $\mathbb{C}$ can be identified with a two-dimensional subfield of $K$, and thus the vector space underlying $Q$ may be regarded as a right complex 4-dimensional vector space (with respect to operations induced from $Q$ ). By definition of the kernel, for any fixed $a \in Q \backslash\{0\}\left(=\mathbb{C}^{4} \backslash\{0\}\right)$ the map

$$
\lambda_{a}: Q \rightarrow Q: x \mapsto a \circ x
$$

is linear with respect to this vector space structure, and $\lambda_{a}$ can be regarded as an element of $G L(4, \mathbb{C})$. We thus obtain a map

$$
\lambda: \mathbb{C}^{4} \backslash\{0\} \rightarrow G L(4, \mathbb{C}): a \mapsto \lambda_{a} .
$$

We shall assume that the unit element 1 of $Q$ is contained in the unit sphere $S^{7}$ of $\mathbb{C}^{4}$. Evaluation at the unit gives $\lambda_{a}(1)=a \circ 1=a$ for all $a$. In other words, the restriction of $\lambda$ to $S^{7}$ would be a cross section for the map

$$
\pi: G L(4, \mathbb{C}) \rightarrow S^{7}: \pi(\gamma)=\frac{\gamma(1)}{|\gamma(1)|} .
$$

The theorem is thus proved by contradiction since actually (as a consequence of $[2,17.1])$ this map does not, in fact, have a cross section. Indeed, choose an ordered orthonormal basis of $\mathbb{C}^{4}$ having 1 as its first vector, and represent elements of $G L(4, \mathbb{C})$ by matrices with respect to this ordered basis. The Gram-Schmidt orthogonalization process yields for every $A \in G L(4, \mathbb{C})$ a decomposition $A=U(A) \cdot D(A)$ with $U(A)$ unitary and $D(A)$ upper triangular with positive real diagonal entries so that, in particular, $D(A) \in \pi^{-1}(1)$. Also $U(A)$ and $D(A)$ depend continuously on $A$. The map $A \mapsto U(A)$ is then a retraction $\varrho: G L(4, \mathbb{C}) \rightarrow U(4, \mathbb{C})$ which respects 
the fibers of $\pi$. Any cross section of $\pi$ would by composition with $\varrho$ yield a cross section of the fibration $\pi \mid U(4, \mathbb{C}): U(4, \mathbb{C}) \rightarrow S^{7}$. But by $[2,17.1]$ such a cross section does not exist.

Proof of Theorem 2. a) It suffices to prove the statement for $N_{\lambda}^{\times}$, since for any 8-dimensional locally compact quasifield $Q$ there is another 8-dimensional locally compact quasifield whose left nucleus is isomorphic to the middle nucleus of $Q$; this may be proved with [4] analogously as in [13, Prop. 4] using the transpose of the translation plane $\mathscr{T}$ coordinatized by $Q$ (cf. $[4, \S 2]$ ). Another quasifield with this property is obtained by recoordinatizing $\mathscr{T}$ with the roles of the first and second coordinate axis interchanged $([5,3.1 .30],[16],[14, \S 7])$.

b) To prove the statement for $N_{\lambda}^{\times}$, consider the map

where

$$
\lambda: \mathbb{R}^{8} \backslash\{0\} \rightarrow G L(8, \mathbb{R}): a \mapsto \lambda_{a}
$$

$$
\lambda_{a}(x)=a \circ x
$$

and its composition

$$
\Delta: \mathbb{R}^{8} \backslash\{0\} \rightarrow \mathbb{R}_{\text {pos }}: a \mapsto \operatorname{det} \lambda_{a}
$$

with the determinant, where $\mathbb{R}_{\text {pos }}$ denotes the multiplicative group of the positive reals. From the definition of $N_{\lambda}$ it is immediate that by restriction $\Delta$ induces a continuous group homomorphism

$$
\delta: N_{\lambda}^{\times} \rightarrow \mathbb{R}_{\text {pos }} .
$$

By $[8,2.3]$ and since $N_{\lambda}$ is closed in $\mathbb{R}^{8}$, we have that $\delta^{-1}\left[r_{1}, r_{2}\right]$ is compact for all $0<r_{1}<r_{2}$. In particular,

$$
F=\delta^{-1}(1)
$$

is a compact normal subgroup of $N_{\lambda}^{\times}$; and since any nontrivial subgroup of $\mathbb{R}_{\text {pos }}$ is cyclic or dense, the image

$$
H=\delta\left(N_{\lambda}^{\times}\right)
$$

of $\delta$ is either 1 ) all of $\mathbb{R}_{\mathrm{pos}}, 2$ ) discrete and isomorphic to $\mathbb{Z}$, or 3 ) trivial.

Therefore all that remains to be shown is that $F$ is finite. For then it is easily seen that there is a closed subgroup $\tilde{H}$ of $N_{\lambda}^{\times}$which is mapped isomorphically onto $H$ by $\delta$. In fact, if $H \cong \mathbb{R}_{\text {pos }}$, take $\tilde{H}$ to be the connected component of 1 in $N_{\lambda}^{\times}$, which by the finiteness of $F$ is a covering group via $\delta$ of the simply connected group $\mathbb{R}_{\text {pos }}$ and thus is isomorphic to $\mathbb{R}_{\text {pos }}$. If $H \cong \mathbb{Z}$, take $\tilde{H}$ to be the subgroup of $N_{\lambda}^{\times}$generated by an element $a$ such that $\delta(a)$ generates $H$. Thus $N_{\lambda}^{\times}$will be a semidirect product of the normal subgroup $F$ and the closed subgroup $\tilde{H}$ isomorphic to either $\mathbb{R}_{\text {pos }}, \mathbb{Z}$ or $\{1\}$.

c) We now prove that the compact subgroup $F$ is finite. Since $N_{\lambda}^{\times}$is a Lie group, it suffices to show that $N_{\lambda}^{\times}$does not contain a torus. Proceeding indirectly, we assume that $N_{\lambda}^{\times}$contains a one-dimensional torus group $T$. The restriction of $\lambda$ to $T$ defines a fixed point free linear representation of $T$ on $\mathbb{R}^{8}$. We may identify $\mathbb{R}^{8}$ as a real vector space with $\mathbb{C}^{4}$ in such a way that $\lambda(T)$ is the group $\Theta$ of dilatations 
by complex numbers of norm 1 . We let $\Theta \subseteq G L(8, \mathbb{R})$ act on $G L(8, \mathbb{R})$ by left translation. Then by the definition of $\left.N_{\lambda}^{\times} \supseteq T\right)$ the restriction of $\lambda$ to the unit sphere $S^{7}$ is $\Theta$-equivariant; and, of course,

$$
\pi: G L(8, \mathbb{R}) \rightarrow S^{7}: \gamma \mapsto \frac{\gamma(1)}{|\gamma(1)|}
$$

is $\Theta$-equivariant too. Therefore there are induced maps $\lambda^{\prime}$ and $\pi^{\prime}$ between the orbit spaces $S^{7} / \Theta=P_{3}(\mathbb{C})$ and $G L(8, \mathbb{R}) / \Theta$ as indicated in the commutative diagram

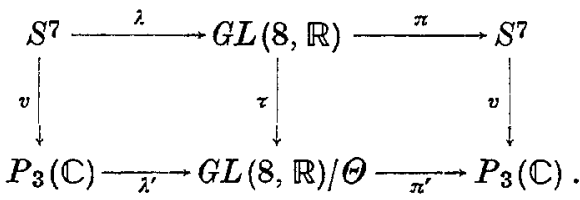

Here $v$ and $\tau$ denote the canonical projections. Since $\pi \circ \lambda=\mathrm{id}$, we have $\pi^{\prime} \circ \lambda^{\prime}=\mathrm{id}$ as well; in other words, $\lambda^{\prime}$ is a cross section for $\pi^{\prime}$. Parts $d$ ) and e) below are now devoted to showing that, in fact, a cross section for $\pi^{\prime}$ does not exist. This contradiction will then prove the theorem.

d) The nonexistence of a cross section for $\pi^{\prime}$. may be seen most quickly by looking at the cohomology groups over the rationals $\mathbb{Q}$ of the spaces involved. In fact, we have $H^{4}\left(P_{3}(\mathbb{C}), \mathbb{Q}\right) \cong \mathbb{Q}$ but $H^{4}(G L(8, \mathbb{R}) / \Theta, \mathbb{Q})=0([7$, p. 90] and [15], [12]).

e) A different proof of this fact making use of the lower homotopy groups of the orbit spaces is the following. We claim first that there exist maps $\eta, \psi, \chi$ making the diagram

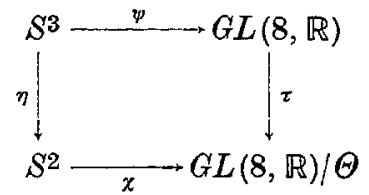

commutative and such that $\psi$ is not null-homotopic.

Indeed, regard $\mathbb{C}$ as a subfield of $\mathbb{H}$, and identify $\mathbb{R}^{8}$ and $\mathbb{H}^{2}$ as real vector spaces in such a way that $\Theta$ is the subgroup of $G L(2, \mathbb{H}) \subseteq G L(8, \mathbb{R})$ given by

$$
\Theta=\left\{\left(\begin{array}{ll}
z & \\
& z
\end{array}\right)|z \in \mathbb{C},| z \mid=1\right\} .
$$

Furthermore, identify $S^{3}$ with the quaternions of norm 1, and define

Via

$$
\psi: S^{3} \rightarrow G L(2, \mathbb{H}) \subseteq G L(8, \mathbb{R}): u \mapsto\left(\begin{array}{ll}
u & \\
& u
\end{array}\right) .
$$

$$
u \mapsto\left(\begin{array}{ll}
u & \\
& 1
\end{array}\right)\left(\begin{array}{rr}
\cos \vartheta & \sin \vartheta \\
-\sin \vartheta & \cos \vartheta
\end{array}\right)\left(\begin{array}{ll}
1 & \\
& u
\end{array}\right)\left(\begin{array}{rr}
\cos \vartheta & -\sin \vartheta \\
\sin \vartheta & \cos \vartheta
\end{array}\right)
$$

$(0 \leqq \vartheta \leqq \pi / 2)$ where $\cos \vartheta$ and $\sin \vartheta$ are considered as quaternions, $\psi$ is homotopic to

$$
u \mapsto\left(\begin{array}{cc}
u^{2} & \\
\cdot & 1
\end{array}\right),
$$


which is not null-homotopic because

$$
u \mapsto\left(\begin{array}{ll}
u & \\
& 1
\end{array}\right)
$$

is a generator of $\pi_{3}(G L(8, \mathbb{R})) \cong \mathbb{Z}([18,23.6$ and 16.7]). Now $\tau \circ \psi$ factors through the orbit space of $S^{3}$ under the group $\{\mathbb{H} \rightarrow \mathbb{H}: x \mapsto z x|z \in \mathbb{C}| z \mid,=1\}$, and this orbit space is $P_{1}(\mathbb{C})=S^{2}$. We define $\eta$ and $\chi$ to be the canonical projection (the Hopf map) and the map induced by $\psi$ respectively.

We now use the maps in diagram (*) to show that a cross section

$$
\lambda^{\prime}: P_{3}(\mathbb{C}) \rightarrow G L(8, \mathbb{R}) / \Theta
$$

for $\pi^{\prime}$ does not exist. From the exact homotopy sequence of $v: S^{7} \rightarrow S^{7} / \Theta=P_{3}(\mathbb{C})$ we have $\pi_{2}\left(P_{3}(\mathbb{C})\right) \cong \mathbb{Z} ;$ from the exact homotopy sequence of $\tau: G L(8, \mathbb{R}) \rightarrow G L(8, \mathbb{R}) / \Theta$ we have $\pi_{2}(G L(8, \mathbb{R}) / \Theta) \cong \mathbb{Z}$, since $\pi_{2}(G L(8, \mathbb{R}))$ is trivial $([18,22.10])$. Consequently, a cross section $\lambda^{\prime}$ for $\pi^{\prime}$ would induce an isomorphism

$$
\lambda_{\sharp}^{\prime}: \pi_{2}\left(P_{3}(\mathbb{C})\right) \rightarrow \pi_{2}(G L(8, \mathbb{R}) / \Theta)
$$

Let $\sigma: S^{2} \rightarrow P_{3}(\mathbb{C})$ be a representative of the homotopy class $\lambda^{\prime}{ }^{-1}[\chi]$ with $\chi$ as in diagram (*). Then in $\pi_{3}(G L(8, \mathbb{R}) / \Theta)$ we have

$$
\tau_{*}[\psi]=[\chi \circ \eta]=\left[\lambda^{\prime} \circ \sigma \circ \eta\right]=\lambda_{*}^{\prime}[\sigma \circ \eta] \text {. }
$$

The left-hand side of this equation is nonzero since $[\psi] \in \pi_{3}(G L(8, \mathbb{R}))$ is nonzero by construction and since $\tau_{\sharp}: \pi_{3}(G L(8, \mathbb{R})) \rightarrow \pi_{3}(G L(8, \mathbb{R}) / \Theta)$ is an isomorphism. The right-hand side, however, is zero since $[\sigma \circ \eta] \in \pi_{3}\left(P_{3}(\mathbb{C})\right)=0$, a contradiction.

Proof of Theorem 3. A closed weak nucleus of an 8-dimensional real division algebra contains the scalar multiples of the unit (since these form the smallest closed subfield of the algebra) and is therefore isomorphic to $\mathbb{R}, \mathbb{C}$ or $\mathbb{H}$ by the Frobenius theorem. We must exclude the quaternions from this list. Proceeding indirectly, we assume that the 8-dimensional real division algebra $D$ has 메 as a closed weak nucleus. Pick any $d \in D \backslash \mathbb{H}$. Then the underlying vector space of $D$ is the direct sum $\mathbb{H}+\mathbb{H} \circ d$; and for all $u, x, y \in \mathbb{H}$ we have

$$
u \circ(x+y \circ d)=u x+(u y) \circ d \text {. }
$$

Thus the restriction of the map

$$
\lambda: D \backslash\{0\} \rightarrow G L(8, \mathbb{R}): a \mapsto \lambda_{a} \text { with } \lambda_{a}(x)=a \circ x
$$

to the 3-sphere $S^{3}$ consisting of the elements of $\mathbb{H} \subseteq D$ of unit norm is described by

$$
\lambda_{u}=\left(\begin{array}{ll}
u & \\
& \\
& u
\end{array}\right) \in G L(2, \mathbb{H}) \subseteq G L(8, \mathbb{R}) \quad(u \in \mathbb{H},|u|=1)
$$

and therefore represents twice a generator of $\pi_{3}(G L(8, \mathbb{R})) \cong \mathbb{Z}([18,23.6])$, cf. part e of the proof of Theorem 2. In particular, $\lambda \mid S^{3}$ would not be null-homotopic. On the other hand, $\lambda \mid S^{3}$ must be null-homotopic as it extends to $D \backslash\{0\}$ and $\pi_{3}(D \backslash\{0\}) \cong$ $\cong \pi_{3}\left(S^{7}\right)=0$. This contradiction proves the theorem. 
The contribution of the second author to this paper is part of his work on a program sponsored by the Deutsche Forschungsgemeinschaft, and he would like to thank the DFG for their support.

\section{Referenees}

[1] J. ANDRÉ, Projektive Ebenen über Fastkörpern. Math. Z. 62, 137-160 (1955).

[2] A. Borel et J.-P. SkRRE, Groupes de Lie et puissances réduites de Steenrod. Amer. J. Math. 75, 409-448 (1953).

[3] T. Buchanan, Finite subgroups of the nuclei of locally compact 8-dimensional quasifields. Preprint, Mathematisches Institut Universität Tübingen 1977.

[4] T. BUCHANAN and H. HäHC, The transposition of locally compact connected translation planes. To appear in J. Geometry.

[5] P. Dembowski, Finite Geometries. Berlin-Heidelberg-New York 1968.

[6] J. Folkman, Equivariant maps of spheres into the classical groups. Mem. Amer. Math. Soc. 95 (1971).

[7] M. GreenberG, Lectures on Algebraic Topology. New York-Amsterdam 1967.

[8] H. $\mathrm{HÄHL}$, Automorphismengruppen von lokalkompakten zusammenhängenden Quasikörpern und Translationsebenen. Geometriae Dedicata 4, 305-321 (1975).

[9] H. H.̈̈L, Geometrisch homogene vierdimensionale reelle Divisionsalgebren. Geometriae Dedicata 4, 333-361 (1975).

[10] D. R. Hugres and F. C. PIPER, Projective Planes. Berlin-Heidelberg-New York 1973.

[11] F. Karscheuer, Die Bestimmung aller stetigen Fastkörper. Abh. Math. Sem. Univ. Hamburg 13, 413-435 (1940).

[12] J.-L. KoszuL, Sur l'homologie des espaces homogènes. C. R. Acad. Sei. Paris 225, 477-479 (1947).

[13] D. M. MaduraM, Transposed translation planes. Proc. Amer. Math. Soc. 53, 265-270 (1975).

[14] H. Satzmans, Topological planes. Advances in Math. 2, 1-60 (1967/68).

[15] H. Samelson, Beiträge zur Topologie der Gruppenmannigfaltigkeiten. Ann. of Math. 42, 1091-1137 (1941).

[16] L. A. Skon Nyakov, Natural domains of Veblen-Wedderburn projective planes. Izv. Akad. Nauk SSSR Ser. Mat. 13, 447-472 (1949); Amer. Math. Soc. Transl. Ser. I 1, 15-50 (1962).

[17] L. A. Skormyakov, Right alternative fields. Izv. Akad. Nauk SSSR Ser. Mat. 15, 177$184(1951)$.

[18] N. Steenrod, The Topology of Fibre Bundles. Princeton 1951.

[19] J. Trrs, Sur les groupes doublement transitifs continus: corrections et compléments. Comment. Math. Helv. 30, 234-240 (1956).

[20] J. A. WoLF, Spaces of Constant Curvature. New York-St. Louis-San Francisco 1967.

Anschrift der Autoren:

Thomas Buchanan

Hermann Hähl

Mathematisches Institut

der Universität Tübingen

Auf der Morgenstelle 10

D.7400 Tübingen 1 